\title{
Gradhiva
}

GRADHIV

Revue d'anthropologie et d'histoire des arts

$9 \mid 2009$

Arts de l'enfance, enfances de l'art

\section{« Dessine-moi un bonhomme ». Universaux et variantes culturelles}

"Draw me a Little Man." Universals and Cultural Variations

\section{René Baldy}

\section{(2) OpenEdition}

\section{Journals}

Édition électronique

URL : http://journals.openedition.org/gradhiva/1432

DOI : 10.4000/gradhiva.1432

ISSN : 1760-849X

Éditeur

Musée du quai Branly Jacques Chirac

\section{Édition imprimée}

Date de publication : 2 septembre 2009

Pagination : 132-151

ISBN : 978-2-35744-009-8

ISSN : 0764-8928

\section{Référence électronique}

René Baldy, « « Dessine-moi un bonhomme ». Universaux et variantes culturelles », Gradhiva [En ligne], 9 | 2009, mis en ligne le 02 septembre 2012, consulté le 30 avril 2019. URL : http://

journals.openedition.org/gradhiva/1432 ; DOI : 10.4000/gradhiva.1432 


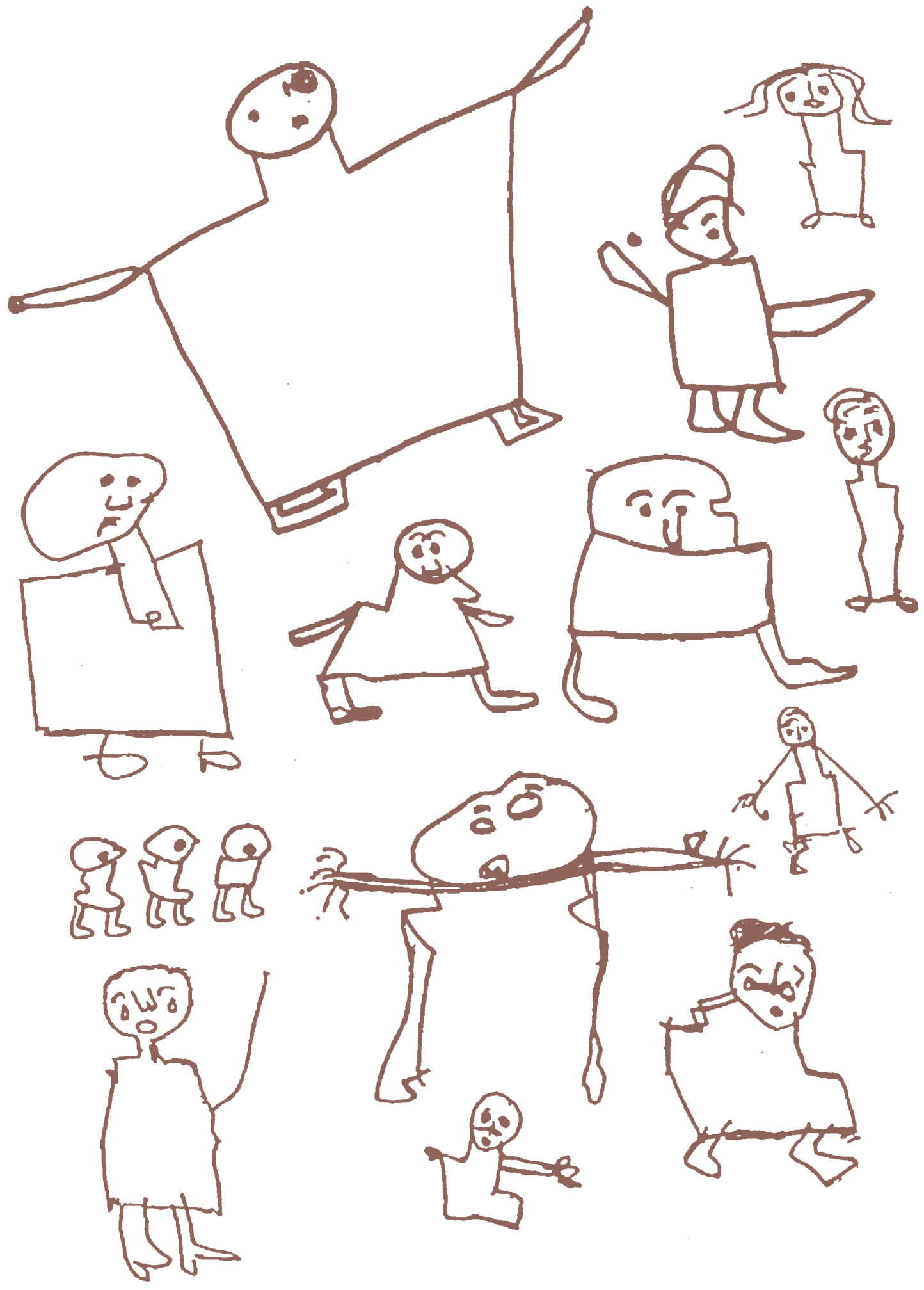




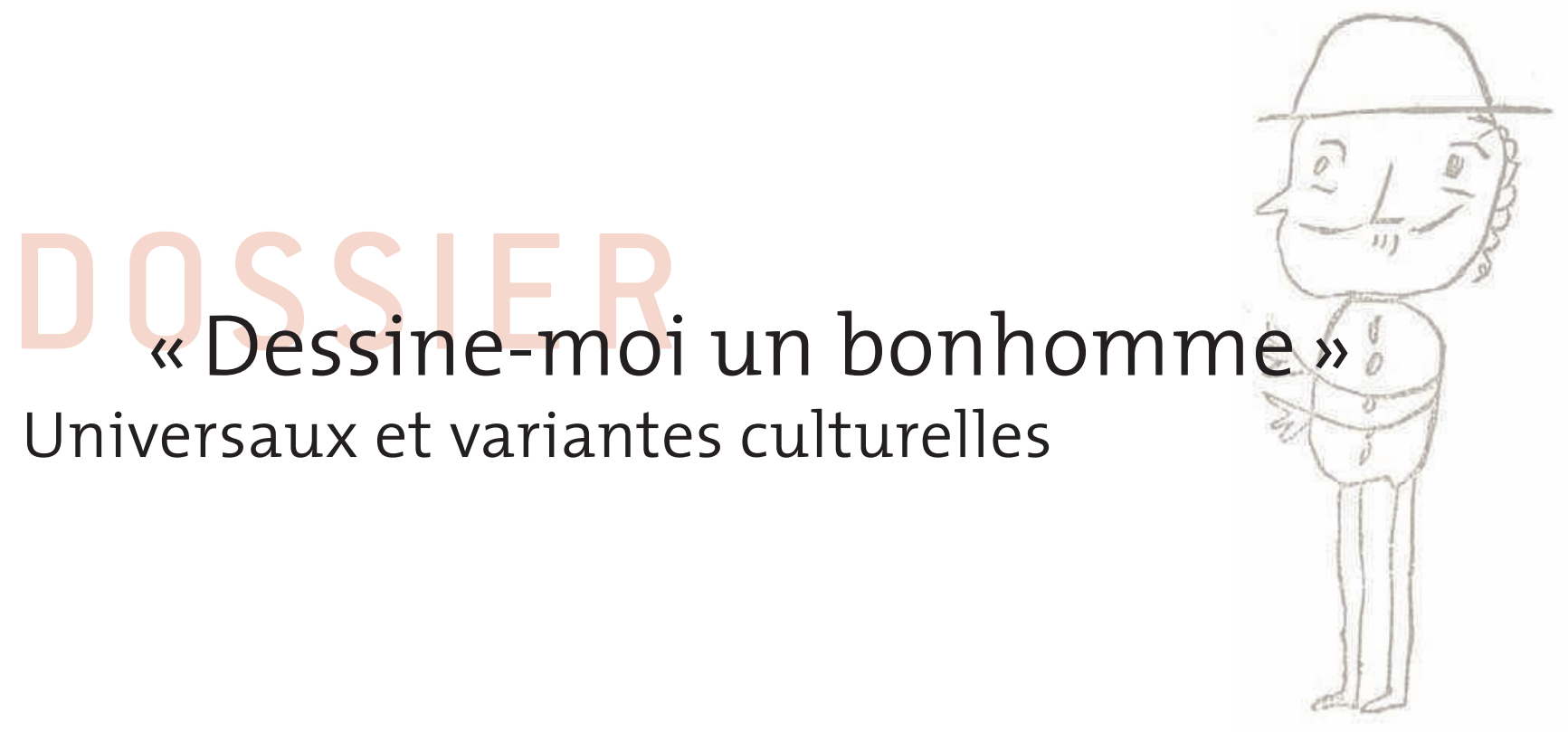

\section{René Baldy}

Fig. 1 Exemples de dessins du bonhomme avec un «torse islamique" (Wilson et Wilson, 1984). Notons l'effort de certains enfants pour lier le tronc et le cou.
Les premiers travaux sur les dessins d'enfance datent de la fin du xix ${ }^{\mathrm{e}}$ siècle. Ricci (1887), Kerschensteiner (1905), Rouma (1912), Luquet (1913; 1927a) ont analysé des dessins d'enfants européens, Paget (1932) et Anastasi et Foley (1936) des dessins d'enfants issus de différentes cultures. Bien que le contexte idéologique ait évolué (Jahoda 1991), les travaux de ce genre n'ont pas cessé. Certes, aujourd'hui, il ne s'agit plus de discuter l'égalité des races ou la théorie darwinienne de l'évolution ni de confondre progression de l'enfant et évolution des cultures "primitives", comme le proposaient Lamprecht (1906) ou Luquet (1927b; 1927c), mais plus précisément d'étudier par le truchement du dessin le développement psychologique. Or, dans les dessins de l'enfant, la personne humaine a toujours occupé une place de choix. Plusieurs auteurs (Cox 1993; Goodnow 1977; Thomas et Silk 1990, etc.) observent que ce dessin-là est probablement aujourd'hui, dans nos sociétés, le plus précoce, le plus fréquent et le plus valorisé par les adultes. C'est aussi le plus étudié par les chercheurs. Aussi consacrerons-nous l'essentiel de notre analyse au dessin du «bonhomme». Nous verrons comment évoluent les compétences graphiques et figuratives des enfants et nous nous interrogerons sur leur universalité dans l'espace et le temps. Les spécificités culturelles observées nous conduiront à une réflexion sur le statut du dessin : est-il un codage réaliste de la réalité ou un langage qui propose une expression graphique culturellement acceptable de celle-ci? Enfin, nous risquerons quelques considérations sur le dessin comme expression de soi et comme construction culturelle.

\section{En Europe aujourd'hui : les phases du bonhomme}

Comment évolue, avec l'âge, la morphologie des bonshommes dessinés aujourd'hui par les enfants européens?

C'est vers l'âge de trois ans que les premiers bonshommes émergent du gribouillage. Le bonhomme rond est composé d'une figure fermée qui se détache du fond et dans laquelle quelques traits s'entrecroisent. Le bonhomme énuméré fait l'inventaire des parties du corps que l'enfant connaît et qu'il envisage l'une après l'autre, parfois en les nommant, comme si le langage oral soutenait l'expression 


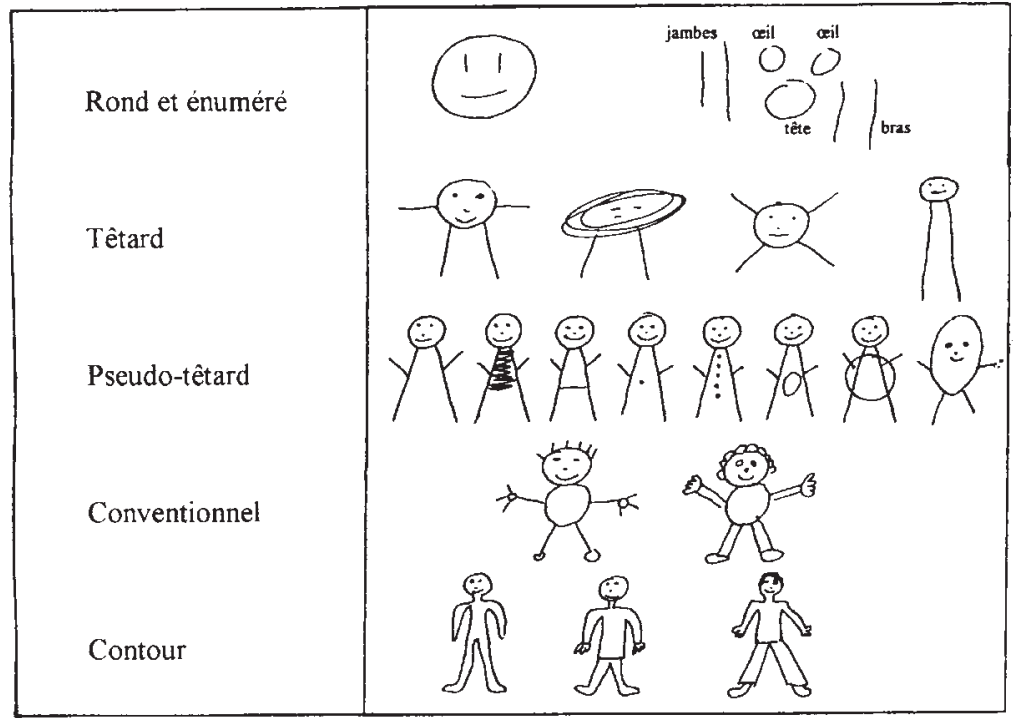

Fig. 2 Exemples de dessins de bonshommes. Source : Baldy 2008. graphique, mais en négligeant leur agencement. Si le bonhomme ovoïde manifeste une vision encore syncrétique du corps humain et le bonhomme énuméré une vision discontinue, les deux témoignent, mais en sens inverse, de l'impossibilité pour l'enfant de coordonner les parties et le tout.

Ces bonshommes rudimentaires progressent vers un bonhomme nommé "têtard", assez mystérieux puisqu'il ne résulte ni d'un apprentissage orienté ni d'une représentation culturelle du corps humain et encore moins d'une expérience visuelle (Baldy 2007; 2008). Il est généralement composé d'une forme ovoïde, contenant les éléments du visage, autour de laquelle rayonnent des traits figurant les membres. Le bonhomme têtard recherche désespérément un tronc comme en témoignent les pseudo-têtards. On pressent sur la troisième ligne de la figure 2 que ces dessins veulent exprimer un bonhomme complet, conventionnel (avec un ventre), mais qu'ils sont encore exécutés selon la procédure du bonhomme têtard : l'enfant dessine ce dernier comme il sait le faire, puis bricole une solution pour figurer le tronc, soit en rattachant les bras aux traits figurant les jambes, soit en comblant le vide situé entre les jambes (espace noirci, trait horizontal, nombril, boutons, etc.), soit en surajoutant un ventre une fois fini le bonhomme têtard. Pour dessiner le bonhomme conventionnel, il faut s'interrompre après le dessin du rond figurant la tête, puis faire un rond pour le tronc et reprendre pour ajouter les membres. Cette opération -interrompre, insérer, reprendre est d'autant plus délicate que la procédure de dessin de la forme rayonnante, souvent répétée, a été automatisée et mémorisée comme un tout. À ce moment du développement, vers quatre ans, on peut penser que la représentation mentale du bonhomme est déjà conventionnelle alors que la procédure d'exécution est encore celle de la forme rayonnante. On retrouve ce retard de l'exécution sur la représentation lorsque l'enfant veut insérer le cou ou des vêtements. Révélateurs de la dynamique du développement, les pseudo-têtards montrent que le bonhomme conventionnel n'émerge pas d'un coup, mais résulte des efforts de l'enfant pour adapter son dessin à de nouvelles exigences figuratives.

Entre quatre et cinq ans, le bonhomme dit conventionnel prend figure humaine. Il résulte de l'addition de parties distinctes verbalisées dont l'exécution n'exige qu'une planification locale : un rond pour la tête, un autre pour le tronc, quatre traits ou «tubes " pour les membres. D'allure géométrique, il est généralement figuré en pied, debout, de face, nu, souvent avec le nombril mais généralement pudique, assez mal proportionné (la tête est exagérée par rapport au tronc) et souriant. Progressivement, le bonhomme s'habille et son identité sexuelle, déjà signifiée par la longueur des cheveux, s'affirme avec les vêtements. Katzaroff notait déjà en 1909 que les garçons ne dessinaient que $1 \%$ de filles et que, réciproquement, les filles avaient tendance à dessiner plus volontiers des personnages féminins. La tendance s'est maintenue, si bien que le dessin du bonhomme témoigne, à cet âge, de la construction de l'identité sexuée de l'enfant. 
À partir de sept ou huit ans, le dessin évolue sous deux aspects. En premier lieu, les signifiants graphiques qui le composent s'efforcent de "rendre" plus fidèlement les apparences visuelles. On voit, par exemple, dans la figure 3, que les signifiants graphiques «nez », «œil » et «bouche » sont géométriques à quatre et cinq ans, puis gagnent régulièrement en complexité et en réalisme au cours du développement.

En deuxième lieu, le bonhomme conçu comme addition de parties fait place au bonhomme contour, qui intègre l'ensemble du corps dans un tout cohérent. L'exécution, planifiée globalement, exige un geste fluide et modulé favorisé par l'apprentissage de l'écriture cursive. Le bonhomme peut désormais se baisser, se mettre de profil, éprouver une émotion et même faire l'objet de manipulations qui sollicitent l'imagination, se métamorphosant, par exemple, en bonhomme-animal.

Bien entendu, cette généalogie abrégée ne rend pas compte de la grande diversité des dessins d'enfants et notre parcours linéaire ne doit pas suggérer des ruptures franches entre un type de dessin et le suivant. La règle est plutôt aux chevauchements. Dans le cas de dessins aussi familiers que celui du bonhomme, l'enfant dispose, à tout moment, d'un schéma routinier pour exprimer sans effort sa vision des choses, et d'un (ou de plusieurs) autre(s) schéma(s) en cours d'élaboration pour expérimenter les nouveaux aspects de sa représentation mentale, de plus en plus riche et complexe et généralement en attente d'une procédure graphique efficace pour l'exprimer. Comme le soulignait déjà Léonard de Vinci, la main a de plus en plus de difficultés à réaliser ce que l'esprit conçoit. On peut voir là l'une des causes de l'abandon, vers la fin de la scolarité élémentaire, du dessin par les enfants.

Cette évolution est-elle générale et intemporelle? L'approche universaliste, d'inspiration rousseauiste, répond oui à cette question. Le développement de la personne en général et celui des capacités graphiques en particulier suivent un

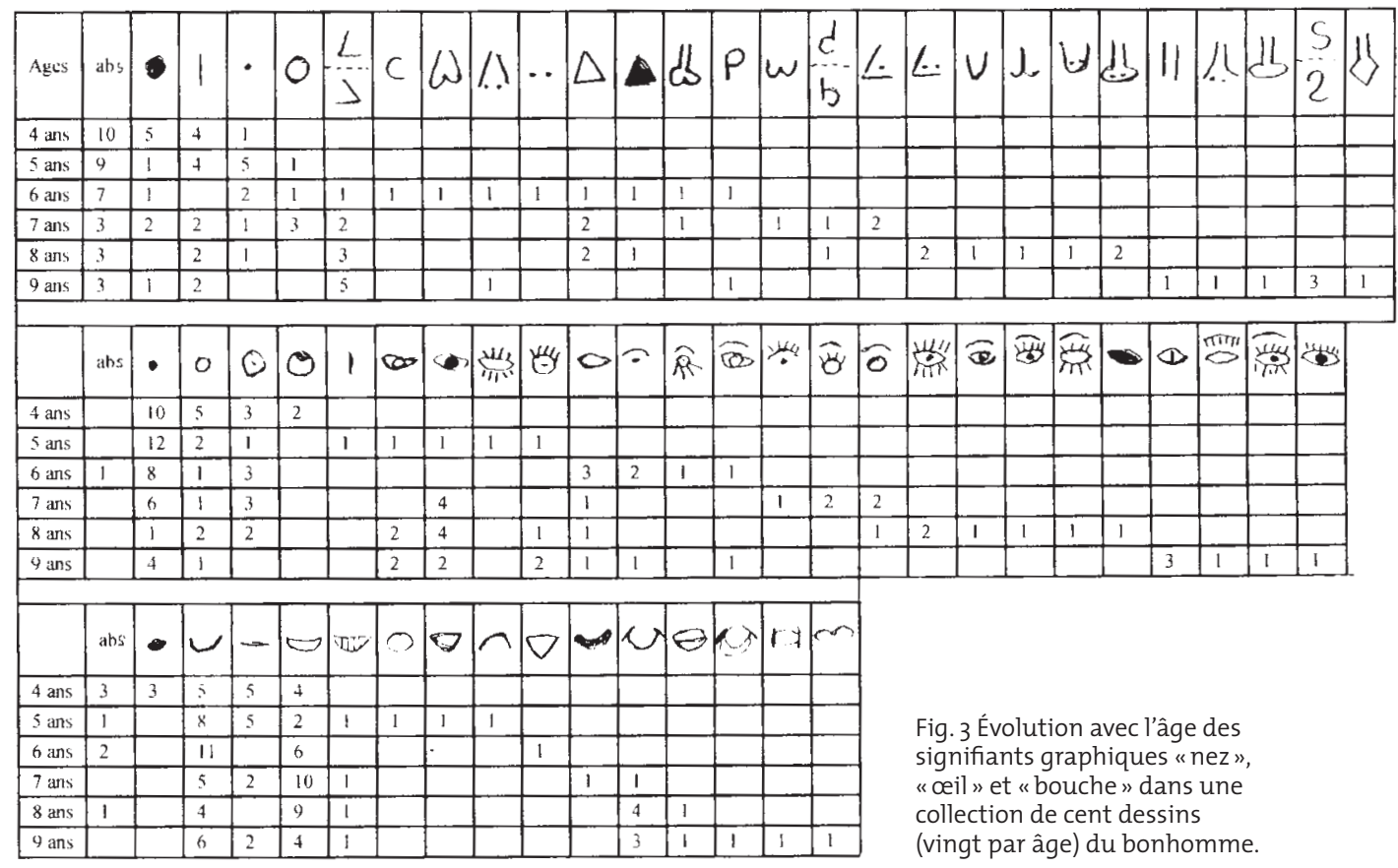


processus endogène "naturel» qui se déroule selon une série de stades indépendants du contexte culturel. L'évolution du dessin de l'enfant a toujours été la même et reste actuellement la même partout. À l'inverse, l'approche culturaliste insiste sur le caractère historiquement daté et culturellement déterminé des représentations humaines et répond non à la question. L'évolution du dessin décrite aujourd'hui dans les sociétés occidentales est spécifique à cette époque et à ces sociétés. Qu'en est-il réellement? La question est probablement plus complexe qu'il n'y paraît, car les comparaisons historiques et interculturelles ne vont pas de soi. Par exemple, les mêmes tâches de dessin proposées à des enfants vivant dans des cultures différentes acquièrent des significations diverses, induisant des attitudes qui ne sont pas équivalentes et comparables. Il est même troublant de se demander ce que pouvait signifier la tâche "Dessine ceci ou cela " pour un enfant interrogé par Paget au début du $x^{e}{ }^{e}$ siècle dans un village africain sans culture graphique. C'est en gardant cette limite à l'esprit que nous poursuivrons l'analyse, en envisageant d'abord les dessins les plus fréquents et les plus aimés dans diverses situations historiques et culturelles.

\section{Des préférences et de leurs contextes}

Les travaux d'Anastasi et Foley (1936), portant sur six cent deux dessins récoltés dans quarante et un pays, mettent en évidence une correspondance entre les thèmes des dessins, les conditions matérielles de l'existence (végétation, animaux, lieu d'habitation, objets courants) et les pratiques culturelles locales (vêtements, activités de travail et de loisir des adultes, religions). D'une façon générale, les éléments dessinés renvoient à l'environnement (neige, mer, arbre, etc.) et les personnages représentés ont des occupations correspondant à la vie commune. Plus récemment, Pruvôt (2005), comparant des dessins d'enfants cubains et français, observe aussi qu'ils reflètent la vie quotidienne et le système de valeurs de la société : "Si vous voulez connaître un pays, faites dessiner ses enfants et écoutez ce qu'ils ont à vous dire " (ibid. : 24). Ainsi, les enfants français expriment un imaginaire influencé par les médias (dessins animés, jeux vidéo, bandes dessinées, films, etc.), alors que les enfants cubains choisissent des thèmes marqués par l'idéologie révolutionnaire (patriotisme, fidélité aux principes marxistes) : "À Cuba, note Pruvôt, les enfants ne dessinent pas le père Noël » (ibid.).

Nous avons souligné que le bonhomme était le thème de dessin préféré des jeunes enfants dans les sociétés occidentales. Cette préférence est-elle intemporelle et universelle? Katzaroff (1909) analysait deux mille six cent soixante dessins libres d'enfants de Suisse romande et, tout en constatant la grande diversité des thèmes abordés, il observait que le dessin du bonhomme arrivait en troisième position, après les objets et les maisons. Paget (1932) disposait de soixante mille dessins imposés (un homme, une femme, un cavalier, une poule) d'enfants de cultures non européennes âgés de cinq à neuf ans dont un grand nombre n'avaient jamais manipulé un crayon et une feuille de papier auparavant. Les observations recueillies l'amenaient à conclure (ibid. : 128) que «le sujet de dessin favori des enfants est la figure humaine". Affirmation que nuancent Anastasi et Foley dans l'étude déjà citée (1936) : selon les auteurs, la présence de personnages dans les dessins d'enfants varie d'une culture à l'autre. Absents des dessins produits par les enfants de Cuba, du Danemark et du Guatemala, ils étaient 
nombreux dans les dessins des enfants mexicains et anglais. Chez ces derniers, ils occupaient une place centrale mais devenaient plus petits, moins discernables dans les dessins des enfants jamaïcains ou néozélandais. Plus récemment, Court (1989) a comparé les dessins de trois groupes d'enfants appartenant à des populations différentes du Kenya : Kamba (agriculteurs), Luo (pêcheurs) et Samburu (éleveurs). Dans un dessin libre, les trois quarts des enfants évitent de dessiner une personne humaine, et, quand ils le font, elle reste petite, peu détaillée et accompagnée d'objets (bateau pour les Luo, vaches pour les Samburu). Dans la tâche "Dessine une vache, une personne et une maison", plus du tiers des enfants luo n'incluent pas de personnage dans leur dessin, et, quand ce personnage est présent, il est très schématique. Enfin, dans la tâche "Dessine-toi en train de manger ", presque tous les enfants se dessinent à table avec d'autres personnes. Prolongeant ces travaux, Aronsson et Andersson (1996) comparent les dessins de trois cent vingt-neuf enfants appartenant à trois cultures qu'ils situent sur un continuum allant de la société traditionnelle à la société moderne : villages de Tanzanie, réfugiés de l'African National Congress (ANC) et villes de Suède. Les dessins doivent illustrer la phrase "Je suis en train de travailler dans ma classe». En Tanzanie, la taille des élèves est beaucoup plus petite que celle du maître, alors qu'en Suède ils sont aussi détaillés, si ce n'est plus. Le maître est figuré plus fréquemment dans une position traditionnelle dans les dessins des enfants africains que dans ceux des enfants suédois (fig. 4).

Dans le même ordre d'idées, Pruvôt (2005) observe que les enfants cubains actuels dessinent des personnages pris dans une activité familiale, alors que les enfants français dessinent plutôt des personnages isolés.

Ces expériences assez hétéroclites permettent au moins de déduire que l'imaginaire des enfants dépend de l'environnement dans lequel ils vivent et que chaque contexte produit des représentations différentes de l'espace social. Une culture rurale (Court 1989), traditionnelle (Aronsson et Andersson 1996), exaltant les valeurs de la collectivité (Pruvôt 2005), induit une représentation du soi immergé dans la vie sociale, alors que la modernité occidentale induit une représentation égocentrée du soi singulier. Les dessins sont donc des indices de la façon dont l'enfant se situe lui-même au sein de l'espace social, dans sa relation à l'adulte, au maître, à la famille.

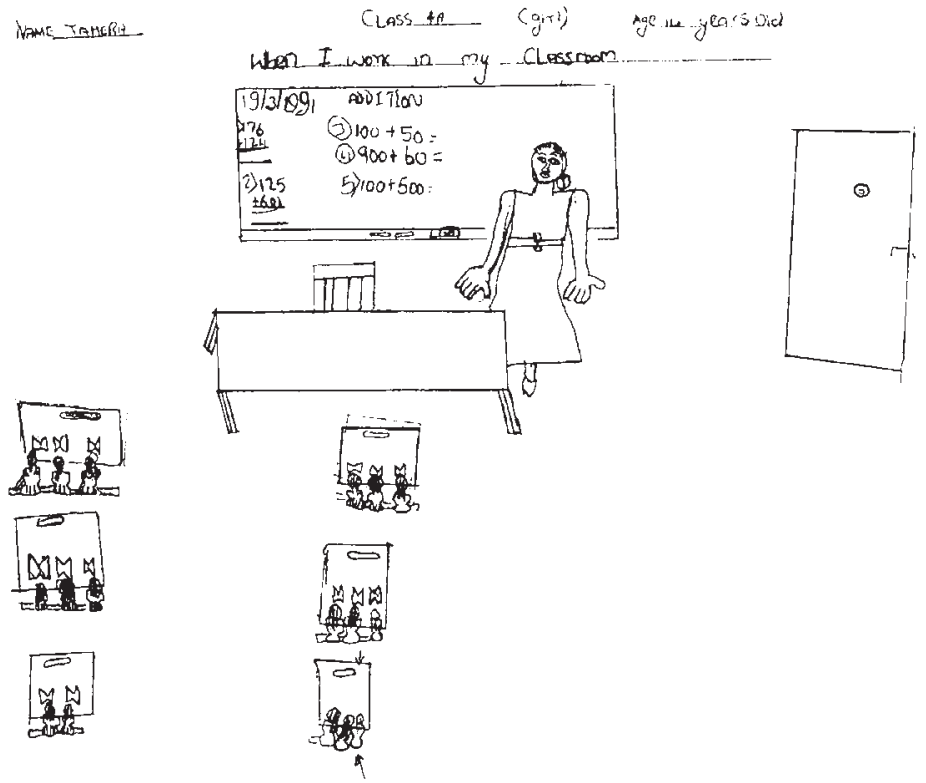

Fig. 4 ( $a$ et b) Dans le dessin du haut, une petite Tanzanienne de 10 ans dessine la maîtresse au centre, dans une position traditionnelle vue de face derrière son bureau et devant le tableau, dans une relation sociale avec l'ensemble de la classe.

Dans le dessin du bas, une petite Suédoise de 10 ans se dessine vue de face, avec beaucoup de détails, au centre de la feuille, dans une position pivot dans l'agencement du dessin. La maîtresse est à l'intérieur de la classe, tendant les bras vers l'élève dans une relation personnelle avec lui.

Source : Aronsson \& Andersson 1996 : 301-314.
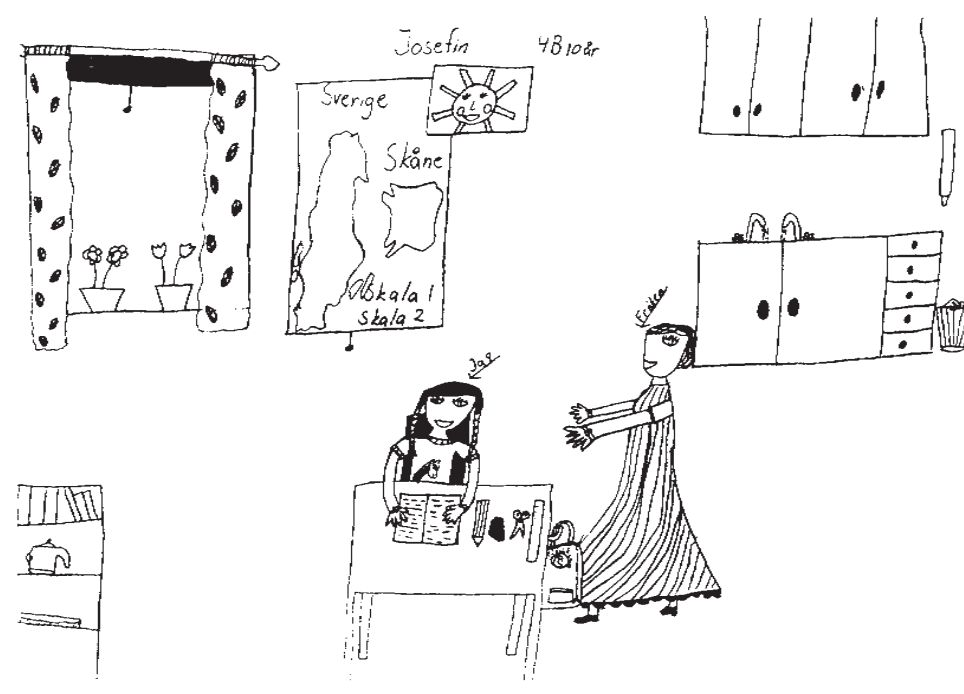


\section{Environnements culturels et propriétés graphiques}

Mais il y a plus. Les personnages dessinés diffèrent aussi quant à leur morphologie. Selon Paget (1932), dans certaines régions d'Afrique, les enfants font des bonshommes délibérément simplifiés, filiformes ou triangulaires. Il observe aussi des bonshommes énumérés, que nous avons décrits comme les premières tentatives d'enfants de trois ou quatre ans, et des bonshommestêtards dont la place du ventre fait l'objet d'un «traitement incertain " (ibid. : 29). Parfois, la tête est petite comme une tête d'épingle et le buste exagérément magnifié, ce qui contraste avec les têtes trop grosses, habituelles dans les dessins occidentaux. Paget souligne au passage un détail révélateur de l'inexpérience graphique de certains enfants : voulant indiquer la couleur de leur peau en ge avec un crayon noir, ils ne peuvent plus figurer les éléments du visage ou remplissant d'emblée leur personnale nombril, ils choisissent alors de les dissocier et les dessinent à l'extérieur du bonhomme.

Plus récemment, Cox, Koyasu, Hiranuma et Perara (2001) puis Nomoto (2007) nous fournissent de beaux exemples de l'influence de la culture sur le jeune dessinateur. Les premiers observent que les enfants japonais dessinent des bonshommes immobiles et vus de face ou de profil, en train de courir, de meilleure qualité que ceux dessinés par des enfants du Royaume-Uni. Ils attribuent cette supériorité à l'influence des bandes dessinées manga sur les petits Japonais. Nomoto, quant à elle, compare les bonshommes dessinés par les enfants français et japonais et leur évolution dans le temps. Les premiers s'attachent à l'effet global de leur dessin et leurs bonshommes respectent de mieux en mieux les proportions, alors que les seconds font preuve d'une attention méticuleuse aux détails et dessinent des bonshommes de plus en plus riches. Ces tendances refléteraient la différence des systèmes éducatifs respectifs. La tradition japonaise privilégie un style de cognition réfléchi et insiste sur l'attention portée aux détails, là où l'éducation française induit un style de cognition plus global et plus "spontané ». Savoir-faire graphique, culture visuelle et normes générales de l'éducation ne sont donc pas uniformes et leurs différences sont très sensibles dans les dessins d'enfants. Partant de là, est-il possible de voir à l'œuvre des «styles» culturels localisés dans l'espace et le temps qui orienteraient dans le détail la forme des bonshommes?

Martlew et Connolly (1996) analysent deux cent quatre-vingt-sept dessins du bonhomme produits par des enfants de dix à quinze ans d'un village de Papouasie-Nouvelle-Guinée sans tradition graphique. Ils observent qu'une proportion importante d'enfants non scolarisés dessine un bonhomme en U inversé, ou "contour ouvert", que les auteurs nomment "Jimi contour", du nom de la vallée où habitent ces enfants. Comme on le voit sur la figure 5a, cette forme, économique sur le plan représentationnel et graphomoteur, capture en un même trait la verticalité et le volume du corps. Quasiment inconnue aujourd'hui en Occident, les auteurs l'identifient comme une variante culturelle. Notons cependant qu'elle est observée en Égypte par Wilson et Wilson (1982a) (voir fig. 7) et en Afrique par Paget (1932). Elle est aussi présente dans les dessins d'enfants de la grotte de Sorèze (voir dessins 12 à 22, p. 158-160).

De son côté, Cox (1998) a étudié les dessins d'enfants warlpiri vivant dans la région de Yuendumu en Australie. La tradition veut que les adultes racontent aux enfants des histoires de voyages et de chasse, qu'ils illustrent en traçant des formes symboliques sur le sable. La personne humaine est représentée assise et vue de dessus, par une forme en fer à cheval. Or, à quatre ou cinq ans, les enfants sont 

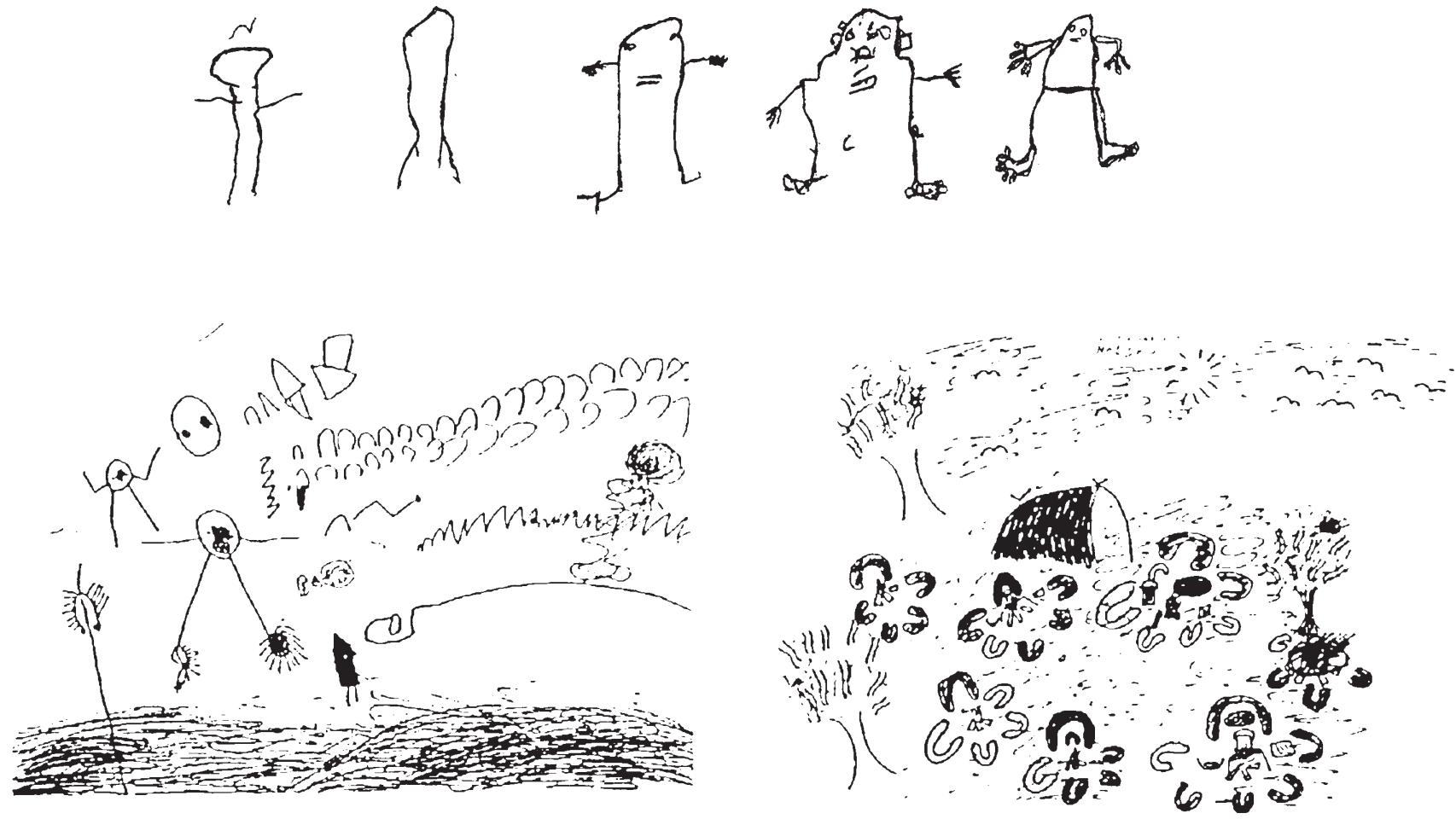

scolarisés et exposés au style de représentation occidental. Ils deviennent donc graphiquement «bilingues» (ibid. : 78), capables de dessiner dans les deux styles (voir fig. 5b). Comme les enfants occidentaux, les enfants warlpiri n'apprennent pas explicitement à dessiner, mais sont influencés par les graphismes disponibles autour d'eux. Dans notre culture, les enfants voient leurs aînés dessiner des personnages en commençant par un rond pour la tête, un autre pour le ventre, etc. Cette forme agit comme un modèle que les enfants s'efforcent de reproduire. De la même façon, la forme en fer à cheval agit comme modèle de représentation des personnes humaines pour les enfants warlpiri. Nous la trouvons moins réaliste que celle que nous utilisons, alors qu'elle est commune dans la culture warlpiri.

Une particularité du torse du bonhomme (fig. 1 p. 132) a été observée par Wilson $(1985)$ et Wilson et Wilson $(1982 a ; 1984)$ chez des enfants égyptiens âgés de neuf à douze ans vivant les uns dans un "village isolé», les autres dans une "ville». Leurs dessins partagent un style commun, dit «égyptien», caractérisé par le torse du bonhomme, rectangle relié à deux segments figurant le cou. Cette forme, peu fréquente dans les pays occidentaux, semble si répandue dans les pays du Moyen-Orient que les auteurs l'ont dénommée «torse islamique ». Il s'agit bien d'un modèle que les enfants égyptiens s'efforcent de reproduire.

Dans le village isolé, le torse islamique correspond au stade final du dessin de personnages. Chez les enfants de la ville, qui ont accès à d'autres modèles, il subsiste, mais plus arrondi et vu de profil.

Focalisons-nous maintenant sur le visage des bonshommes. Dessiné de face ou de profil, il est composé de signifiants graphiques dont nous avons déjà évoqué la diversité. Lorsque le bonhomme est vu de face, le nez, quand il n'est pas tout simplement oublié, est difficile à concevoir graphiquement et donne lieu à une variabilité qu'illustrent les cent quarante-sept formes recensées par Paget (1932).
Fig. 5 ( $a$ et b) En haut, « Jimi contour " produit par des enfants vivant dans un village de Papouasie-Nouvelle-Guinée (Martlew et Connolly 1996). En bas, deux dessins dans un style mixte utilisant des symboles warlpiri et occidentaux (Cox 1998). 


\begin{tabular}{|c|c|c|c|c|c|c|c|}
\hline & 2 & 3 & 4 & 6 & & & \\
\hline A & - .. & $"$ & $\because 0$ & 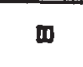 & & - & . \\
\hline B & $n u$ & 0 & $0 \Delta$ & $\omega$ & & & \\
\hline$c$ & $\sim c$ & 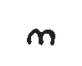 & $m$ m & $\odot$ & & & 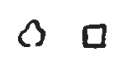 \\
\hline D & 48 & & म & & & & \\
\hline$E$ & $\Delta$ & 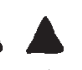 & $\nabla \wedge$ & 1 & & $A$ & \\
\hline & $\Delta \triangle$ & & $\Delta A$ & $\Delta$ & & & \\
\hline & & & $l 2$ & $S$ & & & \\
\hline H & $6 d$ & & $\downarrow \downarrow$ & 1 & & & \\
\hline I & & & to 1 & , & & & \\
\hline $\mathrm{J}$ & $x x$ & $(c)$ & $(2) 62$ & $\partial \int$ & & $d b$ & 26 \\
\hline$K$ & bd U & 11 & ॥ & 14 & $\square$ & ] II & $\tau$ \\
\hline$L$ & هل & ! 6 & $W W$ & $1 U$ & & $a$ & $U$ \\
\hline$M$ & 16 & 0 & $\theta$ & & & & 26 \\
\hline IN & $\omega$ & & & & & & \\
\hline & & & & & & & \\
\hline
\end{tabular}

On peut en déduire que le graphisme «nez» est largement déconnecté de la forme réelle du nez et inspiré de formes communes dans la culture graphique. Par exemple, Paget note que le nez en «double canne » $\boldsymbol{\gamma}$, fréquent en Afrique de l'Ouest, est une de ses formes locales.

Dans leur étude, Wilson et Wilson (1982a; 1984) observent que certains enfants égyptiens du village isolé dessinent fréquemment un bonhomme avec un visage très particulier, figurant un profil concave ou en croissant de lune qui peut présenter deux yeux vus de face (fig. 7).

Ils font dériver ce visage de la forme écrite du chiffre 4. Attesté chez les jeunes villageois (de $25 \%$ à $42 \%$ selon les groupes), il est pratiquement absent chez les citadins. Dans $12 \%$ des cas, ce visage de profil peut présenter deux yeux vus de face. Notons que ce type de profil, en croissant de lune, parfois avec deux yeux, a déjà été observé, mais dans des proportions moindres, par Luquet (1920) sur des bonshommes têtards et par Paget (1932) chez des enfants africains.

La variation culturelle peut être également saisie dans la diachronie, en particulier en Occident, où nous disposons de recherches étalées sur une durée assez longue. Ainsi Wilson et Wilson (1982a), analysant des collections de dessins d'enfants occidentaux d'époques différentes, relèvent deux faits intéressants. D'une part, les pourcentages de dessins du bonhomme de profil diminuent de la fin du xix siècle au milieu du xx ${ }^{\text {e }}$; Sully (1896) en comptait $85 \%$ dans les dessins d'enfants de cinq ou six ans alors que Harris (1963), lors de la révision du test du bonhomme de Goodenough (1926), n'en comptait plus que $28 \%$ dans des dessins d'enfants de huit ans. D'autre part, dans le même temps, les dessins de bonshommes avec un visage de profil et les deux yeux vus de face disparaissent. Ricci (1887) en relevait $70 \%$ dans les dessins d'enfants de six à huit ans à la fin du xix ${ }^{e}$ siècle, alors que Harris n'en comptait plus au milieu du xx $x^{e}$ siècle. Les observations de Kerschensteiner (1905), Rouma (1912) ou Luquet (1920) vont dans le même sens. Par exemple, Luquet observe que, si une majorité des bonshommes têtards ont la tête figurée de face, d'autres présentent le visage de profil avec deux yeux vus de face. Ce type de dessins de bonshommes, qu'il qualifie de "têtards à tête mixte " (ibid. : 688), paraît doublement anachronique : un bonhomme têtard est aujourd'hui dessiné de face, et, quand le bonhomme se met de profil, l'enfant ne dessine qu'un œil. Ainsi, jusqu'au début du xx siècle, le visage de profil avec les deux yeux vus de face pouvait être considéré comme une étape dans le développement du dessin, au même titre que le gribouillage et le bonhomme têtard. Sa disparition progressive au cours du siècle dernier permet aux auteurs de rapprocher les dessins des petits villageois égyptiens de la fin du xx siècle de ceux des petits villageois occidentaux du début. Aujourd'hui, les enfants de neuf ou dix ans peuvent dessiner un bonhomme de profil, parfois avec difficultés, quand on le leur demande ou quand ils expérimentent le personnage en action. En revanche, l'animal est toujours dessiné de profil et avec un seul œil, mais souvent avec deux oreilles et quatre pattes (voir fig. 8).

Mais où est donc passé le fameux profil avec deux yeux vus de face? La question est essentielle étant donné la place que cette figuration a tenu dans les premières analyses du dessin d'enfant. Pour Luquet, ce dessin traduit un mélange de points de vue caractéristique de la phase du réalisme intellectuel, quand l'enfant 
dessine ce qu'il sait de l'objet et non ce qu'il est censé voir. Le passage au profil avec un seul œil serait même un bon indice de la transition vers le réalisme visuel. Aujourd'hui, les enfants dessinent habituellement le bonhomme vu de face, si bien qu'ils disposent d'un schéma routinier qui tend à s'imposer et doit être inhibé pour expérimenter le profil, impliquant donc que l'enfant imagine un nouveau point de vue, découvre de nouveaux signifiants graphiques et mette au point une nouvelle procédure. Cette compétition face/profil peut expliquer le mélange des deux points de vue. La disparition au cours $\mathrm{du} x \mathrm{x}^{\mathrm{e}}$ siècle du visage de profil avec les deux yeux vus de face laisse penser que les enfants d'aujourd'hui seraient de meilleurs inhibiteurs cognitifs que les enfants d'hier. Transition vers plus de réalisme visuel ou inhibition cognitive, de toute façon cette disparition aurait des raisons psychologiques.

À l'inverse, selon l'interprétation culturelle défendue par Wilson et Wilson (1982a), le visage de profil avec les deux yeux vus de face était sous-tendu par des causes externes; s'il a disparu, c'est que ces causes ont cessé d'agir. Jusqu'au début du xx siècle, l'environnement et l'exercice de la compétence graphique restent encore, en Europe, relativement pauvres dans certains milieux ruraux et populaires (peu d'ouvrages illustrés et de publicité, coût élevé du papier et des crayons, etc.). Or, plus les modèles adultes sont rares, plus les dessins des enfants évoluent par influence réciproque et par contagion. Faute de prototypes légitimes, le terrain est favorable à la survivance et à la diffusion de dessins plus ou moins bizarres sortis de l'imagination de certains enfants, qui, de plus, traçaient leurs graffiti sur les murs avec de la craie ou du charbon. Publics, exposés, ces dessins pourraient avoir favorisé la diffusion, voire la généralisation du bonhomme de profil avec les deux yeux vus de face. Dans la collection de dessins de Kerschensteiner, $70 \%$ des enfants qui dessinent un bonhomme avec un tel profil n'avaient aucun livre chez eux. Aujourd'hui, des bonshommes avec le visage de profil et les yeux vus de face naissent probablement ici ou là, mais les conditions culturelles ne sont plus favorables à leur survie et à leur diffusion, étouffés qu'ils sont par toutes les influences graphiques disponibles dans l'environnement.

\section{Mondialisation et autochtonies}

Les bonshommes dessinés par les enfants ne seraient donc uniformes ni dans l'espace ni dans le temps. Cependant, les cultures très contrastées étudiées par Paget ou Anastasi et Foley au début du xx ${ }^{e}$ siècle se sont profondément transformées, perdant une grande part de leurs spécificités. Les modèles graphiques occidentaux, diffusés mondialement, ont étouffé les styles picturaux locaux et tendent à devenir universels. Les travaux plus récents suggèrent cependant que des enfants éduqués dans des cultures distinctes produisent encore des dessins dont les différences sont suffisantes pour en faire des variantes culturelles, parfois incompréhensibles pour qui ne connaît pas le sens des graphismes utilisés.

Ainsi, les travaux de Wilson et Wilson (1984) montrent qu'il existe des dessins des champs et des dessins des villes. Le style graphique des petits villageois égyptiens incorpore de nombreuses formes "primitives " faciles à exécuter (perpendicularité, symétrie, mélange de points de vue, composition spatiale som- 
maire, personnage stéréotypé, etc.) et connaît une évolution limitée, alors que le style graphique des petits citadins traduit un dépassement de ces formes "primitives", avec la découverte de la perspective pour marquer la profondeur et de signifiants graphiques élaborés et variés. De leur côté, les travaux de Martlew et Connolly (1996) montrent que l'habileté à dessiner un bonhomme est influencée par la fréquentation de l'école et plus largement par l'environnement scolaire : les enfants scolarisés dessinent mieux que les enfants non scolarisés (lesquels dessinent des « Jimi contour ") et les enfants non scolarisés vivant dans des villages avec école dessinent mieux que ceux qui vivent dans des villages sans école. L'apprentissage scolaire contribue au développement des capacités de symbolisation, d'analyse et de synthèse, procure des occasions de voir des dessins et de comprendre leur signification, offre des opportunités de dessiner et dispense les encouragements pour progresser. Dans les villages avec école, les enfants non scolarisés qui jouent avec leurs copains scolarisés bénéficient indirectement de ces stimulations. Notons aussi que des enfants sans expérience graphique peuvent, quand on le leur demande, dessiner " directement " un bonhomme conventionnel en exploitant, dans cette tâche nouvelle, leurs capacités générales de symbolisation et leur habileté manuelle. Cependant, comme pour les enfants égyptiens du village isolé, leur façon de dessiner ne dépasse guère les rudiments universels de la figuration graphique. D’une façon générale, les études interculturelles et les quelques comparaisons historiques disponibles suggèrent que des environnements culturels divers peuvent fournir des chemins d'évolution différents aboutissant à d'autres modèles graphiques. Les petits villageois d'Égypte, de Papouasie-Nouvelle-Guinée ou des Warlpiri australiens ne sont pas simplement en retard sur les enfants occidentaux, mais développent des "styles graphiques " distincts dérivant de modèles culturels différents. Ils sont sur des trajectoires développementales plus ou moins divergentes. Comme le soulignait Merleau-Ponty (2001), ce que nous considérons comme le point d'arrivée normal du développement graphique est une réalisation culturelle parmi d'autres.

Qu'en est-il alors de la réflexion, née dans les années 1880 mais approfondie et systématisée par Luquet, sur le dessin comme langage permettant d'exprimer la réalité telle que nous la concevons et comme codage visant à traduire la réalité telle que nous nous la représentons?

\section{Le réalisme au pluriel}

Le dessin comme codage réaliste de la réalité est bien illustré par la description de Luquet (1927a), dont les observations n'ont pas été fondamentalement remises en cause : le dessin est figuratif puisqu'il « a pour rôle essentiel de représenter quelque chose» (ibid. : 99) et il est réaliste "parce qu'il consiste dans la traduction graphique des caractères visuels de l'objet représenté " (ibid. : 100). L'enfant dessinateur élabore un modèle interne, défini comme une "représentation de l'objet à dessiner [...] qui prend nécessairement la forme d'une image visuelle [...] réfraction de l'objet à dessiner à travers l'esprit de l'enfant» (ibid. : 64). Comme on le voit dans la figure $10(\mathrm{~A})$, le modèle interne est une représentation de l'objet, élaborée à partir des connaissances que possède l'enfant et éventuellement de la perception visuelle de l'objet en question. Les connaissances sur les objets du monde se développent, si bien que des modèles internes de plus en plus détaillés sous-tendent des dessins de plus en plus réalistes. 
Luquet définit quatre types de réalisme caractérisés par l'écart entre le dessin et la réalité. Le jeune enfant qui gribouille remarque fortuitement que son tracé ressemble à quelque chose de connu. Le tracé devient alors un dessin, c'est-à-dire un objet symbolique figurant autre chose que lui-même. Dans cette phase, la représentation vient après le tracé. À partir de trois ans, la représentation, soit l'intention de dessiner quelque chose, précède logiquement le tracé. Mais il y a tellement d'obstacles entre l'intention et le résultat que souvent le réalisme est manqué, aussi l'enfant trouve-t-il quelquefois une ressemblance avec un objet tout autre et recycle son dessin. Dès lors, les propriétés du dessin ne sont plus évaluées en fonction de l'intention initiale, mais de "l'intention secondaire " (ibid. : 42), liée à l'interprétation surajoutée. L'enfant peut alors tenter d'améliorer son dessin en s'engageant dans des retouches pour qu'il réponde aux nouvelles exigences. Par ce processus, il apprend à tirer parti de ses «maladresses fécondes »
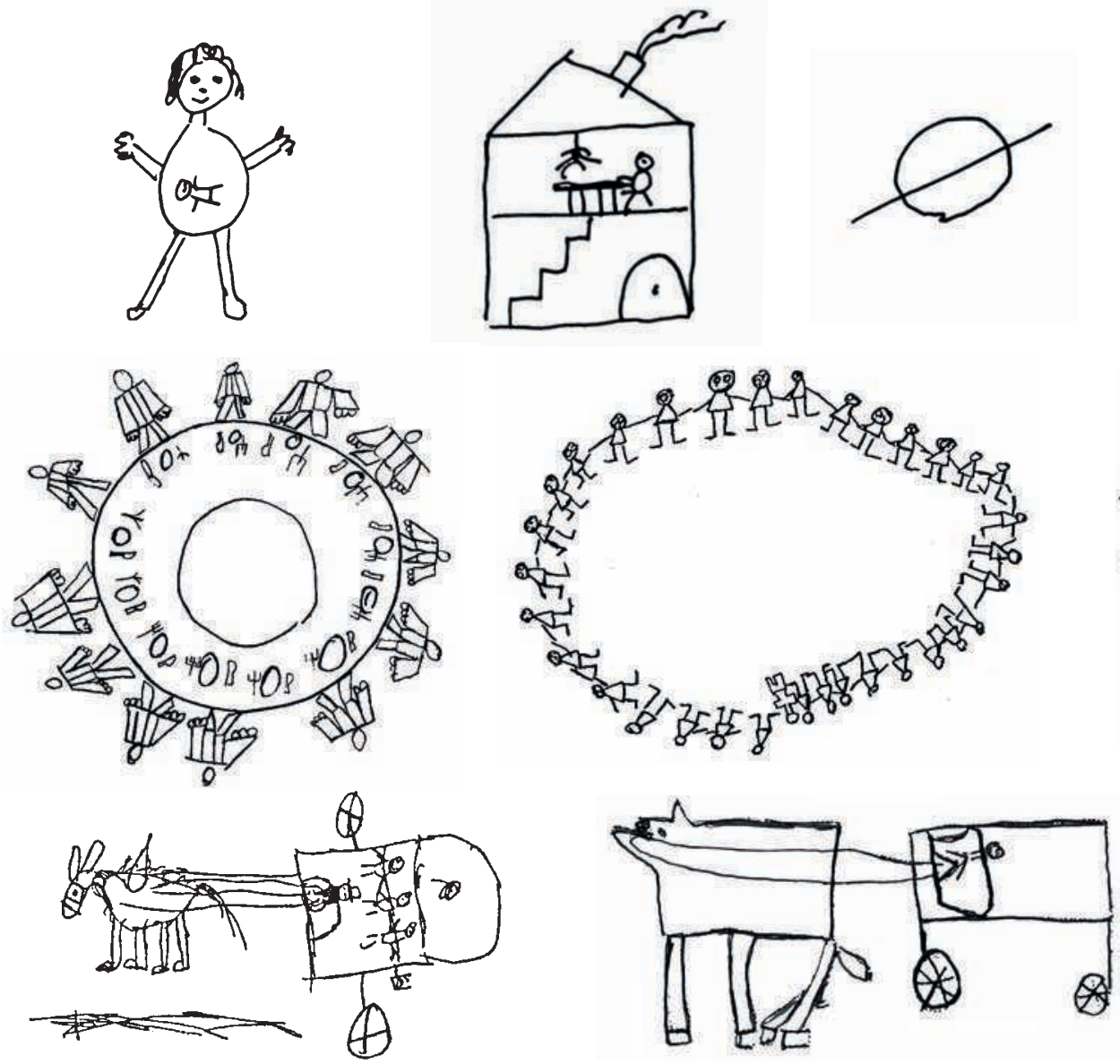

Fig. 8

$1^{\text {re }}$ ligne : transparences : bébé dans le ventre de la maman, intérieur de la maison, pomme traversée par l'aiguille. $2^{\mathrm{e}}$ ligne : rabattements "rayonnant » des convives autour de la table, des petites filles dans la ronde ou des maisons autour de la place, «axial» des arbres le long de la route.

$3^{\mathrm{e}}$ ligne : rabattement du bonhomme "allongé " sur le plateau rectangulaire du chariot vu de dessus et des roues bien rondes de ce dernier (celui de gauche est extrait de Luquet 1927a).

Dernière ligne : le dessin de gauche est une représentation de la Cène du x\|le siècle (extrait de Flocon et Taton 1963) et celui de droite est une représentation de jardin de l'art égyptien (extrait de Panofsky 1975). Nous rapprochons ces dessins sans les confondre, car comme l'ont souligné Francastel (1965) ou Merleau-Ponty (2001), ils témoignent de similitudes de surface entre les dessins d'enfance et l'enfance de l'art.
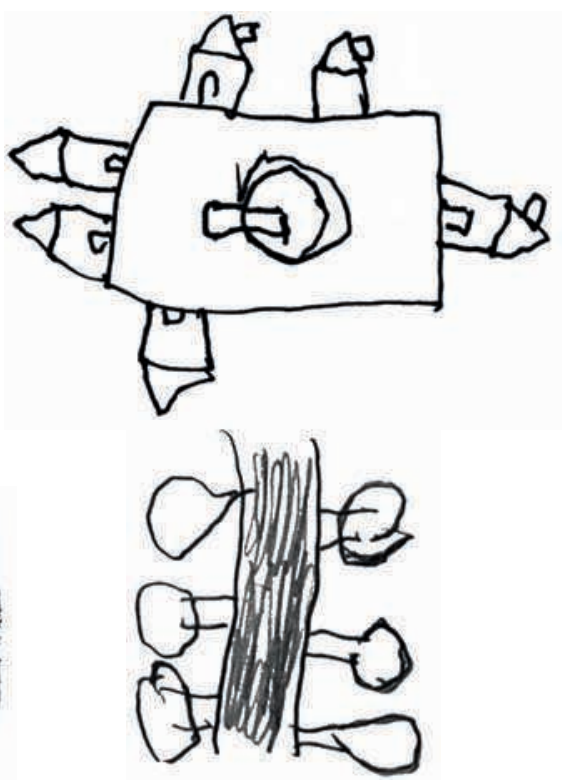
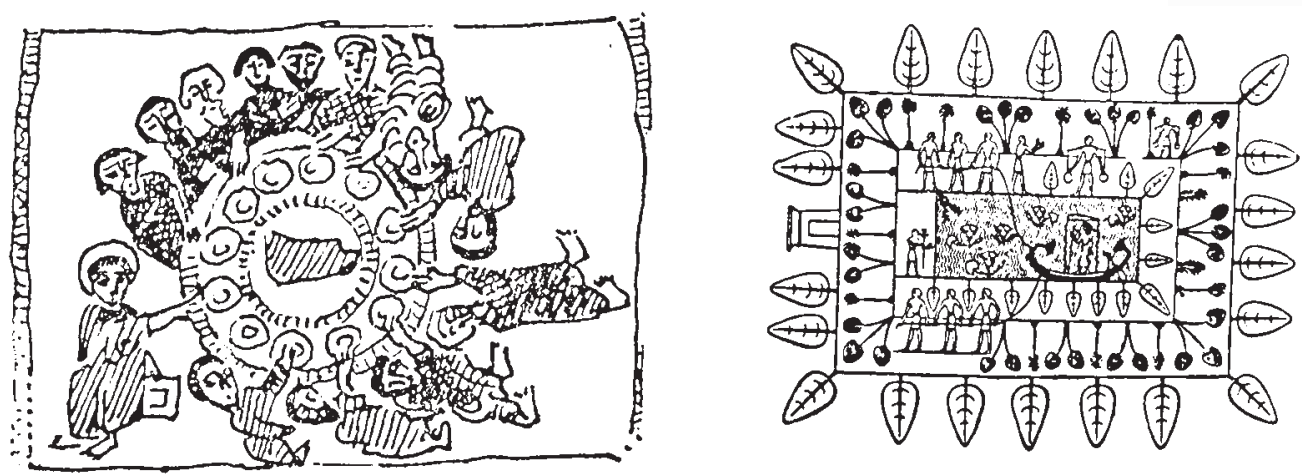
(ibid. : 44). Il peut faire preuve, en toute bonne foi, d'une grande versatilité, le même dessin figurant à tour de rôle une voiture, une maison, etc. À partir de cinq ans, l'enfant inclut dans une synthèse intellectuelle unique ce qu'il voit et ce qu'il sait. Dans cette phase du réalisme intellectuel, « un dessin, pour être ressemblant, doit contenir tous les éléments réels de l'objet, même invisibles soit du point de vue d'où il est envisagé, soit de n'importe quel point de vue, et d'autre part donner à chacun de ces détails sa forme caractéristique, celle qu'exige l'exemplarité » (ibid. : 128). La figure 8 montre que les caractéristiques des dessins répondent à ces exigences. L'invisible y est rendu visible par transparence; certains détails, pour conserver leur forme caractéristique, sont dessinés après rabattement ou plus exactement "pseudo-rabattement", selon l'expression de Piaget et Inhelder (1947), car à cet âge l'enfant est bien incapable de coordonner des points de vue différents. Les dessins témoigneraient donc de transparences volontaires et d'un mélange involontaire de différents points de vue sur l'objet.

Vers neuf ou dix ans, l'enfant prend conscience des «incohérences", "contradictions », «insuffisances» (Luquet 1927a : 153) qui lui échappaient et opte pour le réalisme visuel. Dès lors, il cherche à figurer le monde "comme il le voit». Les transparences intentionnelles disparaissent et une perspective unique tente de remplacer les rabattements. La maison est laborieusement dessinée en perspective et le bonhomme tente de se mettre de profil (Baldy 2008). Luquet définit ce réalisme-là comme «le ferme propos de se conformer à l'apparence visuelle, quand bien même des obstacles divers empêchent cette intention de se réaliser pleinement» (ibid. : 159).

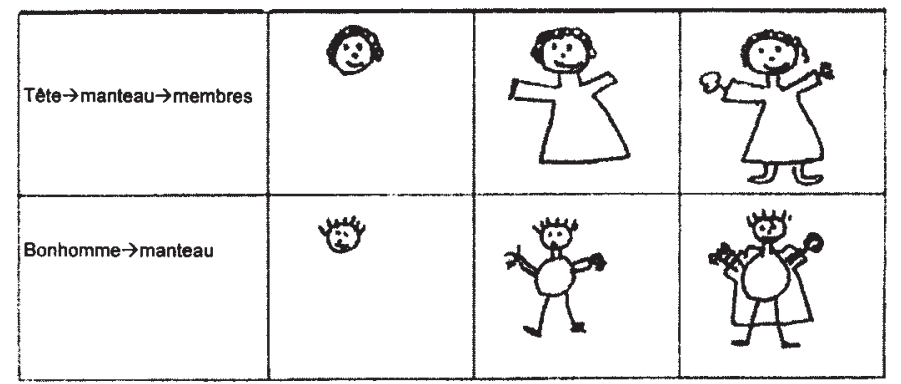

Fig. 9 Procédures d'exécution du dessin d'un bonhomme habillé d'un grand manteau (d'après Baldy 2005).
Un de ces obstacles réside dans la mise au point de procédures d'exécution adéquates. Par exemple, lorsqu'on demande à un enfant de dessiner un bonhomme habillé d'un long manteau, on observe deux procédures d'exécution illustrées dans la figure 9 : la procédure bonhomme $\rightarrow$ manteau (l'enfant dessine le bonhomme comme il a l'habitude de le faire, puis y superpose le dessin des vêtements) conduit inévitablement à des dessins comportant des transparences non intentionnelles; la procédure adéquate tête $\rightarrow$ manteau $\rightarrow$ membres voit sa fréquence augmenter au cours du développement et aboutit à des dessins corrects. Il est intéressant de noter que les enfants de huit ou neuf ans qui utilisent encore la première procédure évaluent les transparences comme des erreurs et tentent de les corriger pour mettre le dessin en accord avec la représentation mentale qui le guide.

Un autre obstacle réside dans l'acquisition des règles et des procédés de la perspective, mais c'est là une autre question.

Dans cette description, les dessins deviennent des transcriptions graphiques de la réalité de plus en plus ressemblantes par rapport aux formes visuellement perçues, c'est-à-dire de plus en plus conformes au réalisme visuel.

\section{Un langage graphique}

Après avoir proposé sa séquence des réalismes, Luquet (1927a), à la fin de son ouvrage, assimile explicitement le dessin à un langage en se demandant «si le réalisme intellectuel ne mérite pas de subsister à côté du réalisme visuel, comme l'arabe à côté de l'anglais » (ibid. : 190). Imitation réaliste ou langage graphique? La 
question ne semble pas tranchée. Pourtant, l'assimilation du dessin à un langage a plusieurs conséquences. D'abord, en ce cas, il est « aussi absurde de demander au dessin de ressembler à la chose dessinée que de demander au mot de ressembler à la chose : ils ne peuvent que signifier, exprimer le monde " (Merleau-Ponty 2001 : 217). Ensuite, l'évolution du dessin ne se décrit pas selon des phases de réalisme se succédant suivant un ordre strict, mais selon des langages qui, au cours du développement, cohabitent dans le répertoire du dessinateur. Tantôt nous dessinons dans une langue, tantôt dans une autre. Enfin, la «réfraction de l'objet à dessiner à travers l'esprit de l'enfant", dont parle Luquet (ibid. : 64), doit transformer, via le répertoire de formes graphiques dont dispose l'enfant, la représentation de l'objet à dessiner en une représentation du dessin de l'objet que l'enfant a l'intention de faire. Comme on le voit sur la figure 10 (B), le modèle interne devient alors un dessin mental plus ou moins schématique composé de formes signifiantes qui seront exprimées graphiquement lors de l'exécution.

Quelle est l'origine des formes graphiques constituant les dessins? D'où tiennent-elles leur pouvoir de figuration? Dans la description de Luquet, elles résultent d'un processus cognitif individuel et tiennent leur pouvoir de figuration de leur «ressemblance» avec les formes visuellement perçues. Les enfants s'efforcent d'abstraire des éléments les formes rendant compte le mieux possible de leurs apparences visuelles. Selon l'approche culturaliste (fig. 10 C), les formes signifiantes sont prélevées dans les modèles graphiques disponibles dans l'environnement et tiennent leur pouvoir de figuration de conventions partagées par les membres d'une communauté. Les dessins produits par ses pairs et ses aînés, les bandes dessinées, les livres illustrés, les dessins animés, les publicités... offrent à l'apprenti dessinateur des occasions de transformer et d'enrichir son répertoire de formes signifiantes. Cependant, l'enfant ne peut assimiler que les signifiants graphiques compatibles avec ses capacités symboliques et graphomotrices. Le développement graphique résulte donc de la mise en tension entre des contraintes intrinsèques à l'acte graphomoteur et la suggestion des modèles culturels offerts par l'environnement. Le poids respectif de ces deux facteurs change au cours du développement. Les signifiants graphiques sont d'abord essentiellement contraints par l'acte de dessiner, puis influencés par l'environnement culturel. Le développement de la compétence graphique ne se réduit donc pas à une ligne évolutive simple.
Fig. 10 Représentation schématique des processus mentaux impliqués dans le dessin selon la description réaliste de Luquet (A), son adaptation (B) et selon l'approche culturelle (C).
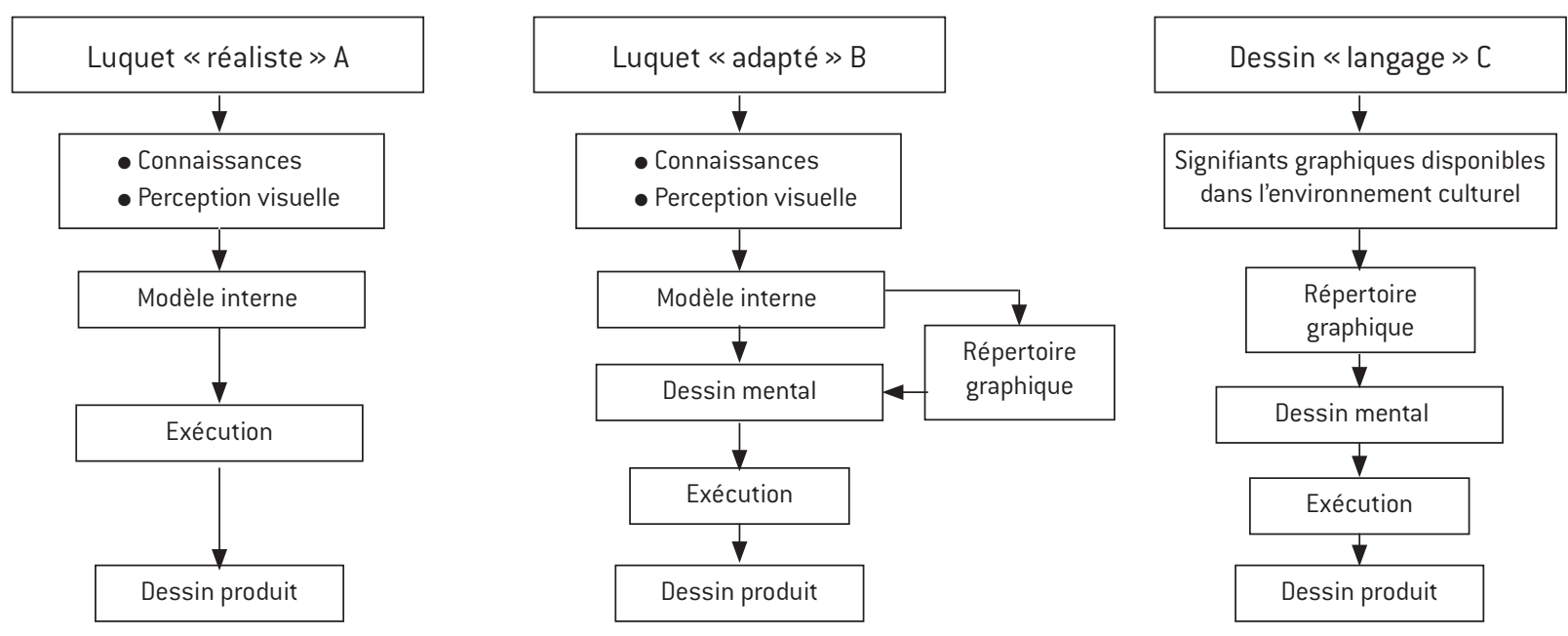
L'existence de gribouillages semblables dans toutes les cultures laisse penser que cette expression est une étape universelle dans le développement du graphisme. Avec l'entraînement, les mouvements impulsifs ralentissent et peuvent être suspendus, si bien que le tracé se nuance pour aboutir aux premiers traits rectilignes et aux premières formes ovoïdes. Ces formes se précisent et s'articulent les unes avec les autres selon des règles simples pour constituer des signifiants graphiques d'ordre supérieur susceptibles d'exprimer un nombre quasi illimité de signifiés. Ronds et traits juxtaposés peuvent figurer le bonhomme énuméré observé dans des cultures et à des époques différentes par Goodenough (1926), Lurçat (1979), Thomas et Silk (1990), Paget (1932) et Cox (1998). L'articulation du rond et des traits donnera la forme rayonnante dont l'enfant usera largement : bonhomme têtard, soleil, fleurs, etc. À ce moment du développement, les enfants commencent à simplifier et symétriser les formes (Pemberton 1990), à orthogonaliser les tracés (Piaget et Inhelder 1947; Wilson et Wilson 1982b), à éviter les intersections (Goodnow 1977), à mélanger les points de vue (Luquet 1927a), etc. La tête du bonhomme est ronde, la maison est carrée, la cheminée est perpendiculaire à son toit pointu, etc. Ces formes, en nombre limité, sont répétées identiques à elles-mêmes d'un dessin à l'autre avec une exécution qui s'automatise. Elles constituent les briques de la fabrication du "dessin mental » encore très schématique que l'enfant exprimera graphiquement lors de l'exécution. Olivier (1974) estime que, vers six ans, ce système de signifiants graphiques est composé d'une trentaine d'éléments, assimilables aux phonèmes d'une langue ou aux graphèmes d'une écriture que l'enfant exploite pour composer ses dessins jusqu'à la fin de la phase du réalisme intellectuel. Parce qu'elles sont le résultat d'une progression autogénérée, ces premières formes graphiques sont universelles (Krampen 1984) et peu sensibles aux influences culturelles. Comme le note Merleau-Ponty (2001) à propos du "polymorphisme enfantin " (ibid. : 177), à cet âge-là, le dessin du jeune enfant exprime sa liberté à l'égard des postulats visuels de notre culture. La théorie universaliste du développement graphique fonde ses arguments sur l'existence de ces ressemblances incontestables entre les dessins des jeunes enfants.

À partir de huit ans, les formes qui caractérisent les premiers dessins sont progressivement relayées par des signifiants graphiques plus sophistiqués qui satisfont les conventions des modèles culturels présents dans l'environnement et qui sont jugés plus ressemblants par rapport à ce qu'ils veulent figurer. Cette transition est un moment critique dans le développement des capacités à dessiner. Les modèles culturels dont l'enfant peut s'inspirer agissent probablement dès le début de l'activité graphique, mais, chez les enfants les plus âgés, ils deviennent déterminants. C'est à travers eux que l'enfant apprend le langage graphique de sa culture. Les suggestions offertes par les modèles culturellement sophistiqués lui permettent de dépasser les caractéristiques universelles des premiers graphismes et de styliser ses dessins. Comme l'ont montré Wilson et Wilson (1982a; 1984) ou Martlew et Connolly (1996), les ressources personnelles de l'enfant s'épuisent lorsqu'elles ne sont pas relayées par les expériences visuelles, graphiques et scolaires. Au cours de son développement, l'enfant dessinateur découvre les modèles graphiques disponibles dans son environnement, se les approprie et les reformule selon ses capacités. Cette reformulation interne des modèles externes approvisionne son imaginaire. 


\section{Expression de soi ou construction culturelle?}

Ces observations nous conduisent à préciser notre position quant à l'éducation artistique, qui fut l'un des terrains sur lesquels l'analyse du dessin d'enfant a débuté et s'est épanouie de manière de plus en plus autonome. Le débat voit s'affronter aujourd'hui deux positions contrastées.

D'un côté, la conception dite rousseauiste de l'éducation artistique postule que chaque individu possède à la naissance le potentiel créateur nécessaire pour inventer seul son langage plastique ou artistique. Le générateur de nouveauté est alors au cœur de la personne. La création est un processus intime, fondé sur une imagination enracinée dans une essence individuelle. Elle reflète l'identité de la personne, elle-même ancrée dans ses caractéristiques innées. Par le dessin, une identité personnelle se projette vers l'extérieur. Protégés des influences néfastes de la société, les enfants préservent et développent ce potentiel. À l'écart de tout dressage, ils peuvent inventer librement des signifiants graphiques originaux pour dessiner le monde. Bref, chacun de nous pourrait créer un langage graphique unique, différent des autres et pourtant compris par tous. Une île déserte, à défaut un village isolé, est un lieu idéal pour l'épanouissement de la création graphique.

Or, à l'inverse, des enquêtes de terrain montrent que, plus les influences culturelles sont restreintes, plus les potentialités créatrices le sont aussi. Les enfants non scolarisés vivant dans des mondes où le répertoire des formes est limité possèdent un langage graphique très réduit et de faibles capacités d'invention. Les enfants scolarisés, habitant dans des zones géographiques foisonnantes de modèles, possèdent un langage graphique riche et qui débouche sur la création. Le générateur de nouveauté est alors dans la culture. Le petit enfant apprend à parler en se référant à un code déjà constitué sur lequel il n'a aucune prise, de même apprend-il à dessiner en se référant également, surtout à partir de sept ou huit ans, à un code qui lui préexiste. Les modèles visuels disponibles portent une structure que l'on peut assimiler à une langue (vocabulaire formel, syntaxe visuelle, conventions graphiques) que l'enfant peut s'approprier pour "bricoler » ses propres créations. Nous sommes tellement habitués à «lire» des images que nous oublions que leur facilité de lecture repose, comme pour le langage oral, sur des conventions apprises dès la petite enfance et partagées par une communauté. L'enfant ne crée pas son langage graphique, pas plus qu'il ne crée son langage oral. Dans les deux cas, il parle la langue de sa culture et même, on l'a vu, de son village s'il est isolé. L'activité artistique est donc enracinée dans les langages collectifs qu'elle ne peut dépasser. Il n'y aurait donc rien de pire pour un artiste en herbe que d'être éduqué sur une île déserte. Tel est l'autre pôle du débat.

En réalité, il nous semble vain d'opposer «expression de soi » et «construction sociale» comme on opposerait "nature» et "culture». Le générateur de nouveauté se trouve probablement dans l'interaction entre les deux. Potentialités individuelles et opportunités culturelles interagissent de façon dynamique. Le développement, graphique ou non, ne peut pas être une aventure individuelle. Dès la naissance et tout au long de sa vie, l'individu construit ses interactions avec le monde qui l'entoure. Comme nous l'avons souligné, aucun enfant n'invente un langage graphique spécifique pour dessiner, pas plus qu'il n'invente un langage verbal pour parler. Dans les deux cas, la créativité artistique d'une personne puise ses origines nécessairement dans les matériaux (langages, modèles, etc.) existant dans sa culture. La singularité individuelle s'insère dans une histoire culturelle. 
L'imagination "pure" ne s'oppose pas à la tyrannie des influences externes. Comme le note Hawkins (2002), c'est à l'intérieur des langages graphiques de sa culture que l'individu peut élaborer son répertoire personnel. L'imagination s'exprime dans cette marge de liberté liée à un certain usage du langage. L'enfant dessinateur n'est sans doute pas un artiste (qui, lui, invente des solutions graphiques nouvelles), mais leurs processus de fabrication mentale et graphique sont proches : l'enfant dessinateur, comme l'artiste, n'est pas ce que l'environnement fait de lui, mais ce qu'il fait de ce que l'environnement fait de lui.

Les observations présentées dans cet article suggèrent trois pistes de réflexion sur l'apprentissage des compétences graphiques. Bien entendu, il ne s'agit pas d'enseigner à l'enfant comment dessiner comme on lui enseigne à écrire. Cependant, comme toute activité, le dessin peut être encouragé, stimulé par la découverte de signifiants graphiques, l'entraînement de la main, le développement des ressources imaginatives. Les dessins d'enfants, souvent affichés sur les murs, sont soumis aux regards et aux commentaires d'autrui. "Il manque le ventre", dira l'adulte devant le dessin d'un bonhomme têtard, commentaire qui participe à l'acquisition des conventions graphiques et amène l'enfant à produire des dessins socialement acceptables. Mais les enfants sont les premiers lecteurs de leurs dessins et, tout comme l'enseignant fait réfléchir ses élèves sur le langage oral, il peut les faire réfléchir sur le langage graphique en leur proposant, par exemple, de faire leur autoportrait et d'inventorier collectivement les différentes formes qui signifient les yeux, le nez, la bouche, mais aussi la chevelure, les mains, etc. Avec cette collection de signifiants graphiques, il est possible de jouer à composer un bonhomme par assemblage : ces cheveux bouclés, ces grands yeux, ce nez, etc. La consultation de bandes dessinées permet d'élargir l'éventail des signifiants. On pourra analyser le dessin d'un enfant: quel est le sexe de ce bonhomme? Comment se tient-il? Quelle émotion semble-t-il éprouver? Les confrontations et les explicitations de ces différentes façons de dessiner permettent la découverte de la variété infinie des sens "qui se cachent » derrière la représentation et favorisent la découverte de signifiants graphiques nouveaux. Les enfants peuvent chercher à indiquer la tristesse, la peur ou la surprise dans le dessin du bonhomme, par exemple (voir Brechet, Picard et Baldy 2007; Picard, Brechet et Baldy 2007). Cette démarche analytique (comme pour l'apprentissage de la lecture) est susceptible de rendre les enfants meilleurs lecteurs de leurs propres œuvres, mais aussi de celles de leurs pairs et, au-delà, de toutes celles qui les entourent.

"L'art se fait avec les mains ", nous rappelle Focillon (1943:112), qui précise un peu plus loin que «la main n'est pas la serve docile de l'esprit» (ibid. : 124). Entre esprit et main, les relations ne sont pas celles de maitre impérieux à servante docile. Léonard de Vinci lui-même nous explique qu'il ne finit pas ses tableaux parce que sa main ne parvient pas à se mettre au niveau de sa pensée. Le dessin de l'enfant unit dans la même origine et le même développement représentation mentale et geste d'exécution. Et, nous pouvons tous en faire l'expérience, la représentation est généralement en attente du geste efficace pour l'exprimer graphiquement. Comme nous l'avons vu, pour que le dessin évolue normalement, la procédure habituelle doit régulièrement être remaniée : pour insérer le ventre du bonhomme têtard, le cou entre la tête et le tronc, les vêtements qui habillent le bonhomme, pour marquer une attitude, une action, etc. D'une façon générale, les modèles proposés par la culture dépassent rapidement les habiletés graphomotrices développées par les enfants et les adolescents, si bien que la divergence entre modèle et production s'accentue, entraînant découragement 

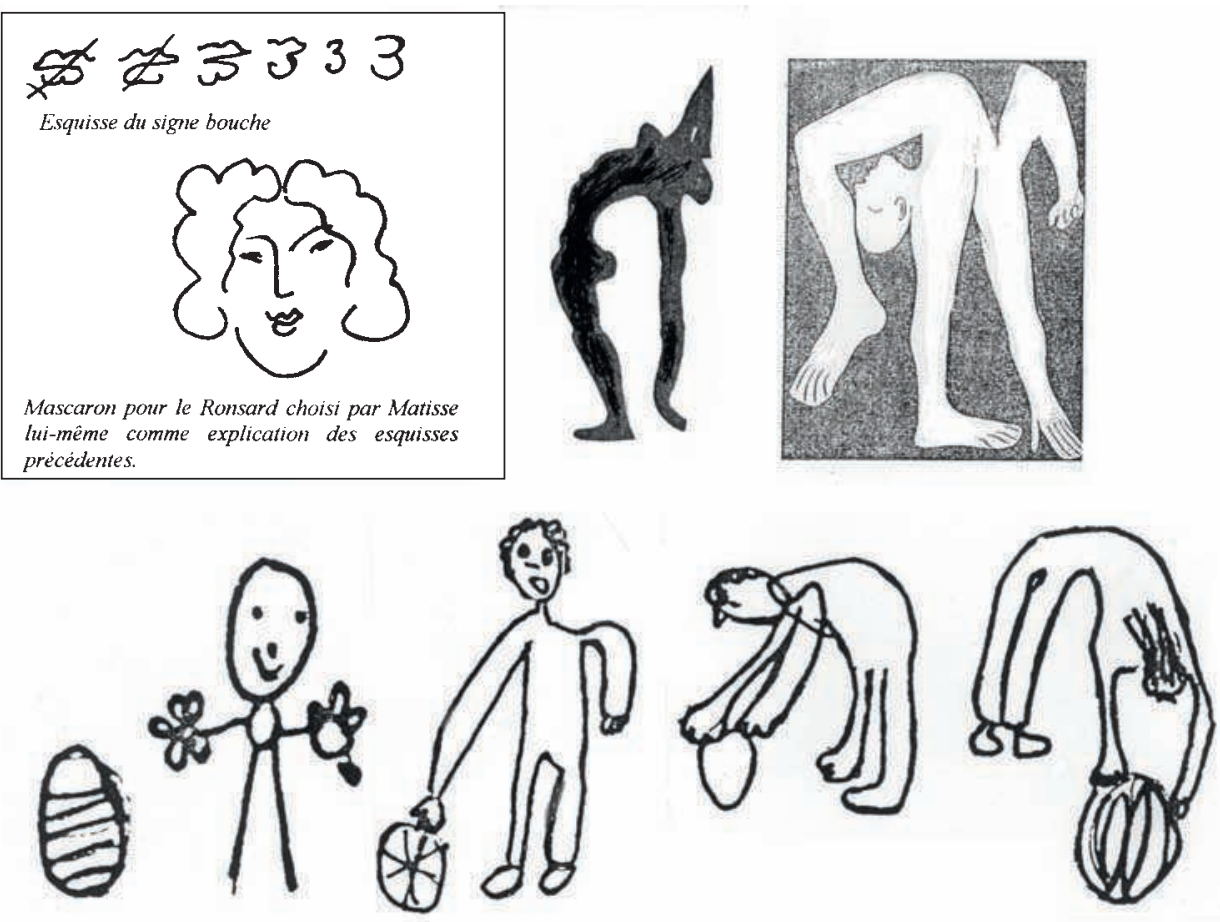

et abandon. Dans le cas de dessins familiers, comme le bonhomme, la répétition de ce geste contribue au caractère stéréotypé du dessin. Comme le notent Cox et Ralph (1996), toutes les occasions de découverte conduisant l'enfant à sortir de ses stéréotypes sont intéressantes, y compris sa confrontation avec les solutions imaginées par Matisse et Picasso (voir fig. 11). Ces exercices invitent l'enfant à sortir de la sécurité du connu, du familier, pour se confronter à l'incertitude, à l'imprévisible, à l'accident, à la maladresse que l'on peut espérer féconde.

Longtemps prisonnier de sa théorie figurative du dessin - le dessin est toujours le dessin de quelque chose : un bonhomme, une maison, un paysage, etc. -, le jeune enfant tarde à concevoir l'idée de création. La description de Luquet n'envisage d'ailleurs pas une phase de dessin créatif. Certes, les dessins de la phase du réalisme intellectuel semblent témoigner d'une grande inventivité, mais les propriétés qui leur confèrent cet aspect naïf, lequel plaît tant à l'adulte d'aujourd'hui, s'imposent à l'enfant et ne résultent pas d'un choix délibéré - le Douanier Rousseau, lui, réélabore ce qu'il sait pour faire comme s'il était un enfant. Progressivement, entre cinq et dix ans, l'enfant change sa théorie du dessin et comprend qu'il peut imaginer et dessiner «quelque chose qui n'existe pas». Il peut alors faire preuve de "flexibilité" représentationnelle et procédurale, selon l'expression de Karmiloff-Smith (1990; 1999). Cette capacité nouvelle émerge lorsque l'enfant peut manipuler ses représentations mentales, qui deviennent comme une matière pour élaborer des objets de pensée nouveaux ne correspondant à aucune réalité perceptive. Dans cette combinatoire créatrice, en dessin aussi une tortue et un lapin peuvent donner naissance à un torpin et une latue.

Université Paul-Valéry Montpellier-III rene-baldy@univ-montp3.fr

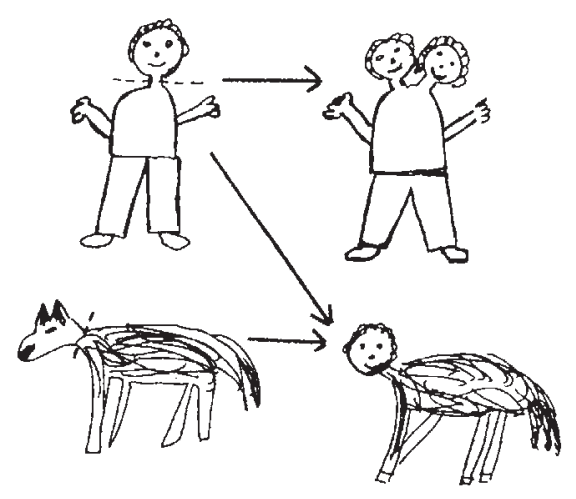

Fig. 11 ( $a$ et b) À gauche, recherche sur le signe "bouche» de Matisse (extrait d'Aragon 1971), acrobates de Matisse et Picasso et dessins d'enfants "dessine un bonhomme qui se baisse pour attraper le ballon ". À droite, on voit que la décomposition de la tête et du tronc du bonhomme autorise le dessin d'un bonhomme à deux têtes. La tête du bonhomme associée à celle de l'animal autorise le dessin d'un être imaginaire avec une tête d'homme et un tronc d'animal.

mots clés / keywords : dessin // drawing • bonhomme // human figure • développement // development • culture // culture. 
Aragon, Louis

1971 Henri Matisse, roman. Paris, Gallimard.

AnAstasi, Anne, et Foley, John P. Jr

1936 "An Analysis of Spontaneous Drawings by Children in Different cultures", Journal of Applied Psychology 20, 6 : 689-726.

Aronsson, Karin, et Andersson, Sven 1996 "Social Scaling in Children's Srawings of Classroom Life: a Cultural Comparative Analysis of Social Scaling in Africa and Sweden", British Journal of Developmental Psychology 14 : 301-314.

\section{BALDY, René}

2005 « Dessins d'enfants et développement cognitif », Enfance $1: 34-44$.

2007 «Pourquoi les enfants de quatre ans dessinent-ils des bonhommes têtards ? » Site internet de la Société française de psychologie. 2008 Dessine-moi un bonhomme. Dessins d'enfants et développement cognitif. Paris, in Press éditions ( $2^{\mathrm{e}}$ édition).

Brechet, Claire, Picard, Delphine, et BALDY, René

2007 «Expression des émotions dans le dessin d'un homme chez l'enfant de 5 à 11 ans », Canadian Journal of Experimental Psychology $6: 141-153$.

\section{COURT, Elsbeth}

1989 "Drawing on Culture: the Influence of Culture on Children's Drawing Performance in Rural Kenya", Journal of Art \& Design Education $8,1: 65-88$

\section{Cox, Maureen V.}

1993 Children's Drawings of the Human Figure. Hove [UK], Lawrence Erlbaum Associates Publishers.

1998 "Drawings of People by Australian Aboriginal Children: the Inter-mixing of Cultural Styles", Journal of Art and \& Design Education $17,1: 71-79$

\section{Cox, Maureen V., et RalPH, Matt Lambon}

1996 "Young Children's Ability to Adapt Their Drawings of the Human Figure", Educational Psychology 16, 3, 245-255.

Cox, Maureen V., Koyasu, M., Hiranuma, H., et Perara, J. 2001 “Children's Human Figure Drawings in the UK and Japan: the Effects of Age, Sex and
Culture", British Journal of Developmental Psychology 19, 2 : 275-292.

Flocon, Albert, et TAton, René 1963 La Perspective. Paris, PUF.

Focilion, Henri

1992 (1943) Vie des formes et Éloge de la main. Paris, Quadrige/PUF.

FRAnCAStel, Pierre

1965 La Réalité figurative. Paris, Denoël/ Gonthier.

\section{Goodenough, Florence}

1926 L'Intelligence d'après le dessin. Le test du bonhomme. Paris, PUF (traduction française, 1957).

Goodnow, Jacqueline J.

1977 Children Drawing. Cambridge, MA, Harvard University Press.

HARRIS, Dale B.

1963 Children's Drawings as Measures of Intellectual Maturity: A Revision and Extension of the Goodenough Draw-man-test. N.Y., Harcourt, Brace and World, Inc.

Hawkins, Bryan

2002 "Children's Drawing, Self Expression, Identity and the Imagination", International Journal of Art Design Education 21, 3 : 209-219.

\section{JAHODA, Gustav}

1991 «Dessins primitifs, dessins d'enfants et la question de l'évolution », Gradhiva 10 : 60-70.

\section{Karmiloff-Smith, Annette}

1990 "Constraints on Representational Change: Evidence from Children's Drawing", Cognition $34: 57-83$.

1999 "Taking Development Seriously", Human Development 42 : 325-32?.

\section{KATZAROFF, M. D}

1909 « Qu'est-ce que les enfants dessinent ? 》, Archives de Psychologie 10, 9 : 125-133.

KeRschensteineR, D.G.

1905 Die Entwicklung der zeichnerischen Begabung. Munich, Gerber.

\section{Krampen, Martin}

1984 "Children's Drawings as Compositions of Graphemes: a Cross-cultural Comparison", Visual Arts Research 10 : 7-12.

\section{LAMPRECHT, Karl}

1906 «Les dessins d'enfants comme source historique », Bulletin de l'Académie royale de Belgique 9 et $10: 457-469$.

\section{Luouet, Georges-Henri}

1913 Les Dessins d'un enfant. Paris, Alcan, «Bibliothèque de philosophie ».

1920 « Les bonhommes têtards dans le dessin enfantin », Journal de psychologie normale et pathologique : 684-710.

1927a Le Dessin enfantin. Paris, Delachaux et Niestlé.

1927b « Le réalisme intellectuel dans l'art primitif (I) », Journal de psychologie normale et pathologique 30 : 888-927.

1927c « Le réalisme intellectuel dans l'art primitif (II) », Journal de psychologie normale et pathologique 30 : ?65-79?

\section{LURÇAT, Liliane}

1979 L'Activité graphique à l'école maternelle. Paris, Les Éditions ESF.

Martlew, Margaret, et ConnolLy, Kevin J.

1996 "Human Figure Drawings by Schooled and Unschooled Children in Papua New Guinea", Child Development 67 : 2743-2762.

\section{Merleau-Ponty, Maurice}

2001 Psychologie et pédagogie de l'enfant. Paris, Verdier.

\section{Noмото, Tomoko}

2007 Étude du développement cognitif du traitement des propriétés d'objet : apports de la figure complexe de Rey $B$. Thèse de doctorat de psychologie, Paris. Université Paris-VIII.

\section{Olivier, Fernand}

1974 «Le dessin enfantin est-il une écriture ? 》, Enfance 3, 5 : 183-216.

PAget, G.

1932 "Some Drawings of Men and Women Made by Children of Certain Non-European Races", Journal of the Royal Anthropological Institute of Great Britain and Ireland 62 : 127-144.

\section{PANOFSKY, Erwin}

1975 La Perspective comme forme symbolique et autres essais. Paris, Éditions de Minuit. 
Pemberton, Elizabeth

1990 "Systematic Errors in Children's

Drawings", Cognitive Development 5 : 395-404.

Piaget, Jean, et InHelder, Barbel

1947 La Représentation de l'espace chez

l'enfant. Paris, PUF.

PicARD, Delphine, Brechet, Claire, et BALDY, René

2007 "Expressive Strategies in Drawing Are

Related to Age and Topic", Journal of Nonverbal

Behavior 3 : 243-25?.

PRUVÔT, $M$. V.

2005 « Le dessin libre et le dessin de la famille chez l'enfant cubain. Étude comparative avec un groupe d'écoliers français »,

Pratiques psychologiques $11: 15-27$.

\section{Ricci, Corrado}

1887 L'arte del bambino. Bologne, Zanichelli.

Rouma, Georges

1912 Le Langage graphique de l'enfant.

Bruxelles, Misch et Thron.

\section{SulLy, James}

1896 Studies of Childhood. Londres, Longmans, Green.

Thomas, Glin V., et Silk, Angèle M. J. 1990 An Introduction to the Psychology of Children's Drawing. Londres, Harvester Wheatsheaf.

WiLson, Brent

1985 "The Artistic Tower of Babel: Inextricable Links Between Culture and Graphic

Development", Visual Arts Research 11 : 90-104.
Wilson, Marjorie, et WiLson, Brent

1982a "The Case of the Disappearing Two-eyed Profile: or How Little Children Influence the Drawing of Little Children", Review of Research in Visual Arts Education 15 : 19-32.

\section{WiLson, Brent, et WiLson, Marjorie} 1982b "The Persistence of the Perpendicular Principle: Why, When and Where Innate Factors Determine the Nature of Drawings", Review of Research in Visual Arts Education 15 : 1-18.

1984 "Children's Drawings in Egypt: Cultural Style Acquisition as Graphic Development", Visual Arts Research 10 : 13-26.

\section{Résumé / Abstract}

René Baldy, «Dessine-moi un bonhomme». Universaux et variantes culturelles. - Dans ce texte, nous analysons l'évolution avec l'âge des bonshommes dessinés par des enfants européens et nous questionnons l'universalité de ce dessin. Nous confrontons l'approche universaliste, pour laquelle le développement des capacités graphiques suit un processus endogène indépendant de la culture, et l'approche culturaliste, qui insiste sur le caractère historiquement daté et culturellement déterminé des représentations humaines. Les spécificités culturelles observées [thèmes des dessins, particularités graphiques] suggèrent que des enfants de cultures différentes produisent des dessins dont les spécificités sont parfois suffisantes pour en faire des variantes culturelles incompréhensibles au lecteur qui ne connaît pas les symboles utilisés. Nous nous interrogeons sur le statut du dessin comme codage réaliste ou langage graphique et, pour terminer, nous risquerons quelques implications pédagogiques.
René Baldy, "Draw me a Little Man." Universals and Cultural Variations. - In this text, we analyse how the human figures drawn by European children develop with age and we question the universality of this type of drawing. We confront the universalist approach, for which the development of graphic capacities follows an endogenous process independent of culture, with the culturalist approach, which insists on the historically contingent and culturally determined character of human representations. The cultural specificities observed (themes of drawings, graphic details] suggest that children from different cultures produce drawings with specific features which are sometimes sufficient to make them cultural variants incomprehensible to the viewer who is unfamiliar with the symbols used. We discuss the status of drawing as realist coding or graphic language and finally, we venture some consideration of pedagogical implications. 